\title{
Machining Distortion Analysis of Aerospace Components Using the Contour Method
}

\author{
Mats Werke $^{\mathrm{a} *}$, Mosharraf Hossain ${ }^{\mathrm{b}}$, Daniel Semere ${ }^{\mathrm{b}}$, Anders Wretland ${ }^{\mathrm{c}}$ \\ ${ }^{a}$ RISE IVF AB, Box 104, 43122 Mölndal, Sweden \\ ${ }^{b}$ KTH Industrial Production, Brinellvägen 68, 10044 Stockholm \\ ${ }^{c}$ GKN Aerospace AB, Flygmotorvägen 1, 46181 Trollhättan, Sweden \\ *Corresponding author: mats.werke@ri.se
}

\begin{abstract}
During machining the accumulated residual bulk stresses induced by previous shape forming process steps, such as forging, casting or additive manufacturing and subsequent heat treatment will be released. This may cause undesirable geometric distortions of the final component and thereby high rejection rates and costs. This problem can be reduced by adjusting process- and design parameters. This paper presents a methodology for minimizing machining distortions. The methodology is based on a combination of procedures for prediction of machining distortions, using the Contour method and procedures for adjustment of machining distortions. Practical experiences are discussed and demonstrated using an aerospace component. The methodology should be executed in close cooperation between several actors in the value chain and best results may be achieved by combining several concepts for adjustment of machining distortions. Further research in conjunction with the Contour method, adaptive fixturing and toolpath adjustment is recommended.
\end{abstract}

Keywords: Machining distortions, Contour method, Adaptive Fixtures, NC-Code optimization

\section{Introduction}

During machining the accumulated bulk stresses induced by previous shape forming process steps, such as forging, casting or additive manufacturing and subsequent heat treatment, will be released. This may cause undesirable residual deformations and geometry errors of the final component. The distortions are difficult to predict and in practice, therefore wide tolerances and several machining steps are applied to avoid reprocessing or, at worst, scrap. By taking into account the residual stresses during process planning a significant improvement in dimensional accuracy can be achieved. This may save millions of Euros in scrap costs and correction procedures for the manufacturing industry [1].

This paper proposes a methodology for minimizing such distortions that emerges during machining, to be used in order to facilitate a minimum of deviations from the nominal shape of the final product. The methodology is based on a combination of procedures for prediction of residual stresses in the work piece, prediction of deformations in the component during material removal and procedures for minimizing the distortions.

The overall methodology is described in Section 3 whereas procedures for residual stress prediction and mitigation during subsequent material removal operations is described in Section 4, supported by a test case, a forged aerospace component. Different concepts for adjusting machining distortions are described in Section 5 and conclusions are summarized in Section 6 together with suggested issues for further research.

\section{Related Work}

Several investigations have been reported in the literature on machining and residual stresses. The majority of these are focusing on the residual stresses induced from the machining operations. However, relatively few papers have addressed distortions coming from the redistribution of the initial residual stresses after the material removal. Distortions in machining of aerospace parts are often attributed to the redistribution of the residual stresses given all other operation parameters are optimized for adequacy [2]. The existing research literatures refer to the prediction and measurements of the initial residual stress field as well as estimating the distortions and proposing solutions to counter these distortions to attain the tolerances.

Earlier investigations concerning how to measure residual stresses using both non-destructive or destructive techniques such as X-ray diffraction and hole drilling have been reported. The limitations to these methods in terms of depth of measurement is well known. In this paper, the Contour Method [3][4], which is an accurate method that overcomes these limitations has been used. The method has been validated by a combined experimental and computational analysis as reported in an earlier work by Werke et al in [5]. This paper is based on these findings. 
Redesign of parts and considerations during process planning, particularly machining sequence and fixture configurations, are proposed in some papers to comply the desired tolerance [6][7]. Cerutti et. al. discussed the effect of positioning the workpiece with some offset, a parameter numerically optimized to find the position where the stress distribution assumes its minimum [8]. Chantzis et.al. presented a work with similar approaches and also provide a simulation based work flow to find the optimized offset for a minimum distortions in [9]. These works specifically refer to conditions where the stress profile fits to the machining task. Therefore, it is difficult to generalize for other stress profiles.

This paper proposes a new method for adjustment of the NCcode in order to compensate for the distortions during the manufacturing process.

\section{Methodology}

The first step is to predict the work piece bulk stresses induced prior to machining, see fig. 1.

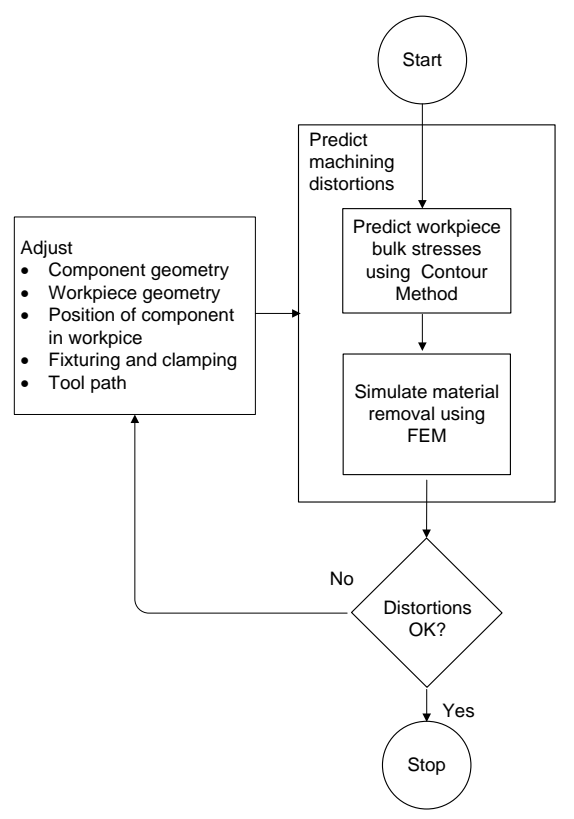

Figure 1 Overall methodology

This is performed using the Contour method. This method uses the fact that a body that contains residual stresses will deform when the body is cut into sections. The tractions required to restore the deformed section to its original shape are equivalent to the out of plane residual stress released by sectioning, see fig. 2.
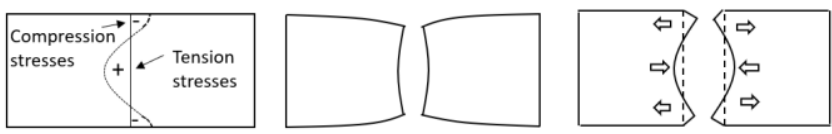

Figure 2 Schematic illustration of Contour method [3][4]

This information is used as input data to the second step, a material removal simulation for calculation of the distortions. If the distortions exceed the requirements from the customer, then adjustments should be performed until the level of distortions are within tolerances. The methodology is described below in conjunction to a test case, an aerospace component.

\section{Prediction of machining distortions}

An aerospace component in a nickel based alloy was used as test case, see fig. 3. The component was analyzed in its net shape condition before machining where after the deviations from its nominal geometry was verified. The work piece was hot forged in $1100 \mathrm{C}$ with a screw press followed by solution annealing and then machining in several setups to final form. The geometrical shape had a tendency to drift out of tolerance and twist around the axial direction. The twist was max 0.51 $\mathrm{mm}$ and $\mathrm{min}-0.69 \mathrm{~mm}$. The ambition was to set up a model for analysis and adjustments of the drift.
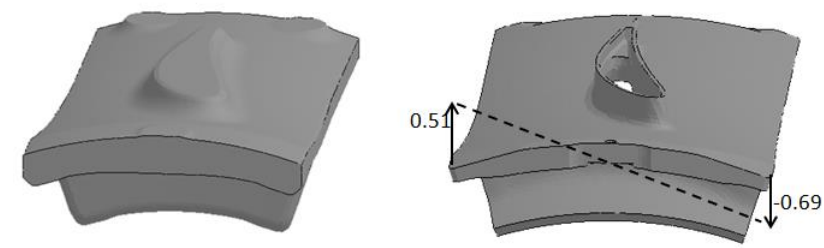

Figure 3 Workpiece before and after machining and illustrated twist

\subsection{Prediction of workpiece bulk stresses}

Predict stresses in cut sections: The work piece was cut in 5 sections $(0,50,90,120,240 \mathrm{~mm})$ according to fig. 4, using Wire Electro Discharge Machining (W-EDM). The cut section displacements were then measured using Geometric Optical Measurement (GOM) technique. The GOM measurement requires the cut surfaces to be made with lowest possible surface roughness, to make it possible to detect the subtle deviations from an ideal flat shape after the parting is performed. Thus, the choice of a precision machining method that is able to cut along a straight line while securely clamped is crucial.

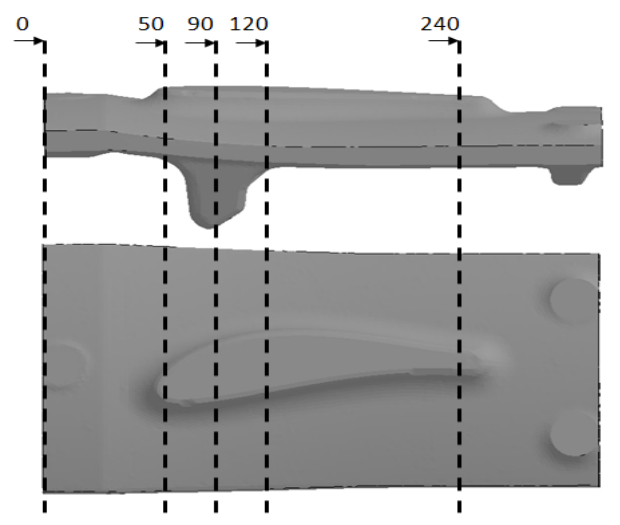

Figure 4 Workpiece cut in 5 sections

In the test case this procedure was carefully planned where the work piece was prepared with start and finish holes for 
the EDM wire, to enable lowest possible deflection of the component during each parting operation, see fig. 5. Thus, the component was actually functioning as its own fixture during the major part of each parting operation.

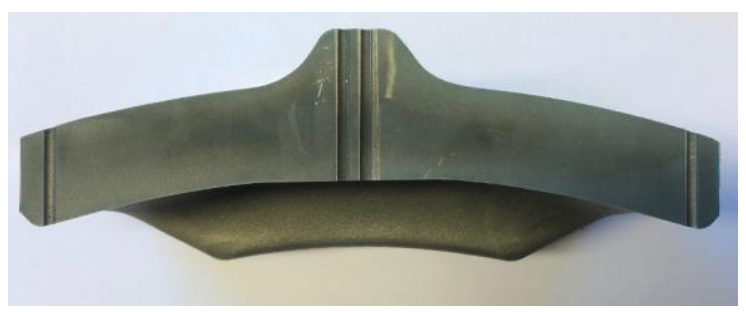

Figure 5 Cut section with four drilled holes for guidance of EDM

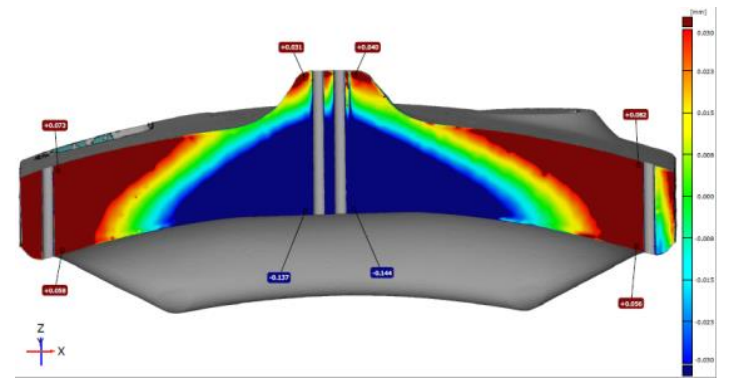

Figure 6 GOM measurement of cut section

The results from GOM according to fig 6, were translated to Finite element models of the sectioned parts. The out of plane distortions in the cut sections are directly related to the out of plane stresses before sectioning and were applied as boundary conditions to finite element models of the parts after sectioning. The displacement surfaces from opposite sides of the cut were averaged in order to improve the accuracy of the results. The out of plane stresses (x-stresses) were then calculated according to fig. 7 .

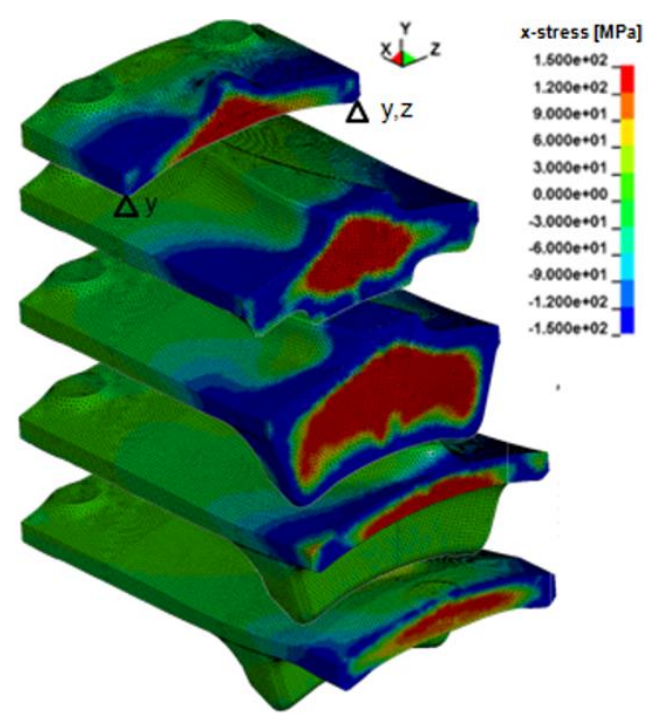

Figure 7 FE-models of the sectioned workpieces with applied $x$-displacements and resulting $x$-stresses
Calculate work piece stresses: One limitation, using the Contour method, is that the calculated stresses are limited to the out of plane stresses from the cut sections. The method may be expanded to a 3-dimensional stress state using multiple cuts at 90 degrees [10] or 45 degrees from the first direction [11]. Thus, substantial work is needed in order to predict the complete stress field on a component, using multiple cuts. In order to simplify the stress analysis a numerical iterative approach using FEM for prediction of 3dimensional stresses based on single cut measurements was developed and tested according to the following.

The calculated out of plane stresses (x-stresses) in the cut sections were mapped onto the work piece mesh and interpolated between the sections. After mapping and interpolation, a spring back calculation was performed which, due to equilibrium, established a 3-dimensional stress condition. This redistribution of stresses caused the originally mapped out of plane stresses to decrease. To overcome this problem an iterative procedure with remapping of the out of plane stresses on the work piece with subsequent spring back calculations was performed. The procedure was repeated until the out of plane stresses reached its original level and resulted in a more correct 3-dimensional stress state [5].

\subsection{Simulation of material removal}

A simplified approach for simulation material removal was utilized, where material was instantaneously removed using linear FEA. The mesh of the removed material was deleted and a spring back analysis of the remaining component was performed according to fig. 8 .

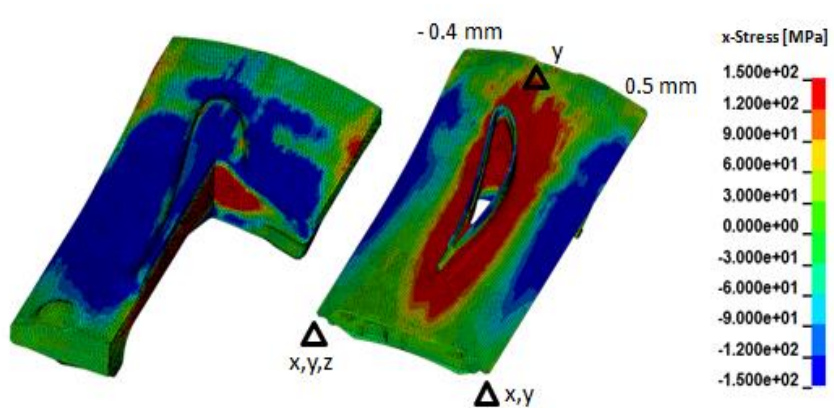

Figure 8 Stresses and distortions after material removal

Correlation between the calculated results and measurements of the top surface of the component using a conventional Coordinate Measurement Machine (CMM) show that the calculation is in good agreement with the appearance of the real part, see fig. 9 . 


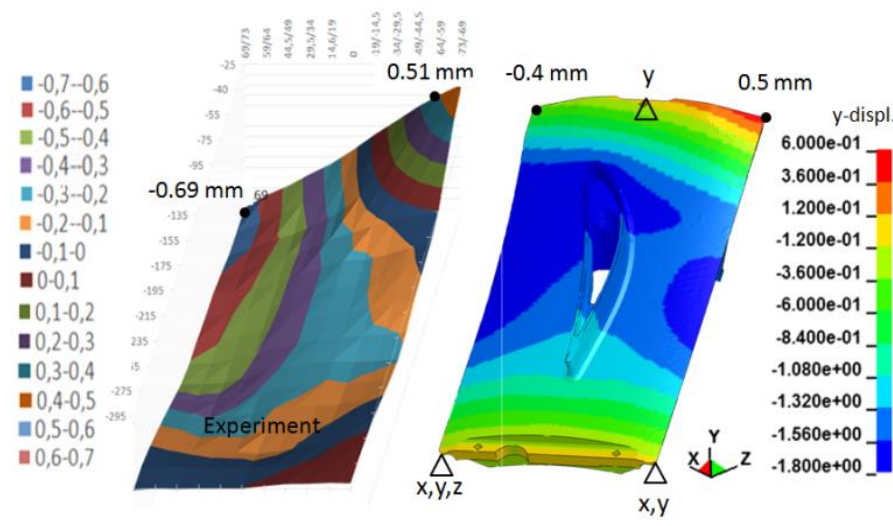

Figure 9 CMM measurement at the top surface (left) compared to calculated deformations (right).

Several concepts for how to handle geometry deviations caused when inherent bulk stresses are released during subsequent material removal are described below. They all assume the existence of a billet, in which the inherent stress field from previous forming and heat treatment operations is well known. Thus, the accurate stress field will act as the foundation for further advanced analysis of how the billet behaves during the following steps of material removal. Further, additional benefit can be achieved from accurate simulations of the intended actions of how to handle distortions most efficiently.

\subsection{Adjust component position in work piece}

The level and distribution of the residual stresses that are released in the component during the material removal process depends on where the final component is positioned in the billet. To move the part in the billet can be most effective if the blank and the final component have relatively simple geometries. Thus, one possibility to minimize for distortion is to virtually investigate the effects on part geometry while changing the position of the final part relative to the blank. Thereby the volume in the billet with the least effect on geometry deviations of the final part can be determined, see fig. 10 .
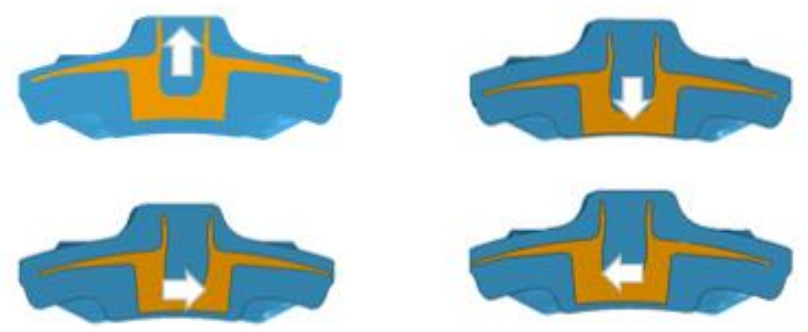

Figure 10 Adjustment of position of the component relative to workpiece

\subsection{Adjust work piece and component geometry}

Another approach is to adjust the design of the component and redistribute the process induced stress field. This is illustrated in fig. 11 where a stiffness bar is included in the component in order to minimize deflections due to internal stresses. Even though this approach, that from practical reasons is a less likely choice for an already existing design, it is an approach much similar to topological design optimization including residual stresses. As such it might be able to not only change the geometry of the work piece and thereby redistribute and reduce the stress field but also be able to contribute to weight optimization.
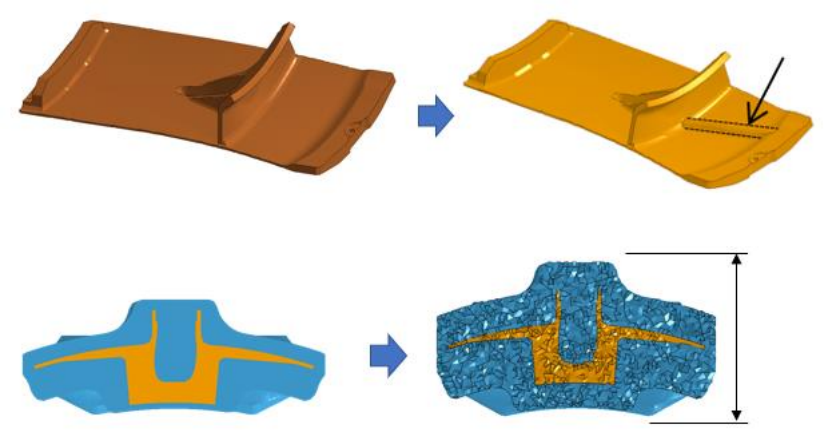

Figure 11 Adjustment of component- and workpiece geometry

\subsection{Adaptive fixtures}

During machining, the work piece must be held and locked to a fixed position in the workspace of the machine tool. This is normally done by use of some kind of fixture or clamping device. The upside of this concept is that the position of the part is well known. The downside on the other hand is that the clamps at the same time may prevent the part from needed relaxation. This may introduce new stresses due to the changed stress state which may affect the geometrical stability of the part and cause unexpected distortions during material removal operation. The preferred solution would therefore be a clamping device that could be adaptive and provide some dimensional flexibility.

The general requirements for an adaptive and reconfigurable fixture set-up have been discussed by several researchers. A comprehensive list of work performed in this area is compiled by Borboni et al [12] whereas more specific applications with respect to aero engines are discussed by Papastathis et al [13].

Distortions during material removal are largely dependent on the continuous change of inherent phenomena in the work piece, such as changes in the residual stress state due to material removal. It is therefore important to enable the part to relax, i.e. regain a state free from the distortions imposed because of the relieved internal stresses. This is ideally done continuously, but in practice at reasonably frequent instances, most often related to by "best practice".

Thus, the interface between the part and the clamping device should, to some degree, be flexible in order to accommodate for distortions that inevitably will be the result of released stresses. However, this flexibility must be exercised such that the dynamic stiffness of the ongoing material removal process can be maintained. This is of course of particular importance when high and intermittent process forces are applied, such as during conventional milling and even more so during milling with super abrasives such as ceramic or Cubic Boron Nitride (CBN) tools. Flexibility to enable a rigid 
enough clamping of the part must therefore be exercised in steps - between the material removal operations, rather than continuously during the operations.

The most direct and simple form of adaptive clamping is therefore to just release and immediately clamp the part again.

This concept requires that the interface elements are "floating" to regain the stiffness requirements as just discussed. A number of practical solutions to this problem has been discussed and also practically tested in the European collaborative project INTEFIX [14].

Because of the many parameters to set during the design of a fixture, the release and clamp concept ha to be validated first using simulation. Furthermore, simulation enables the optimal amount of material removed in the release and clamp operation and also offers support when defining the most effective position of each clamping device.

Thus, with support from a FE-model, it is possible to calculate the required clamping position and the corresponding force, introduced at each clamping interface. In the following, a calculation procedure using several steps of material removal is demonstrated in conjunction to the test case according to fig. 12. The calculation indicated the need for high clamping forces during the first material removal steps (node 3, 4, 7 $10)$, whereas the required clamping forces during the second steps were low, see fig. 13. The proposed calculation procedures may be used for virtual test of various clamping options. Further, to adapt the clamping to the geometry variation during machining and in addition minimize the stresses introduced by clamping.
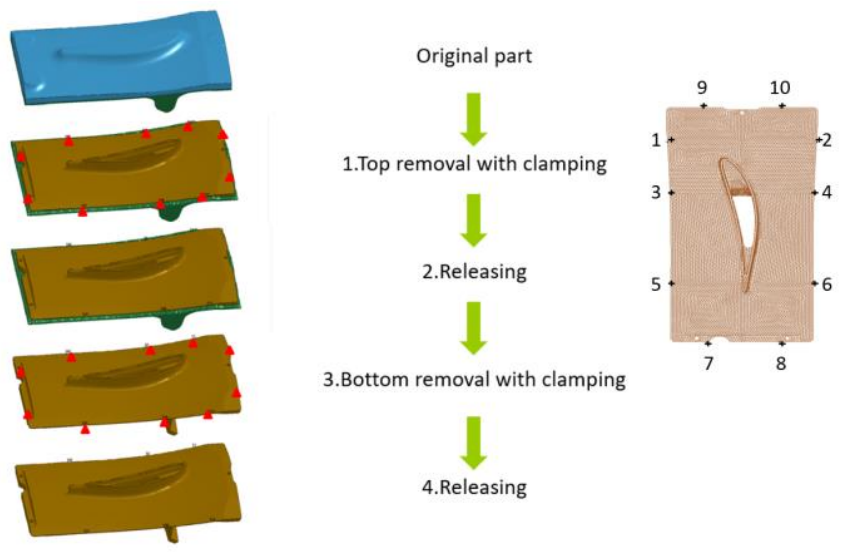

Figure 12 Material removal in two steps including clamping

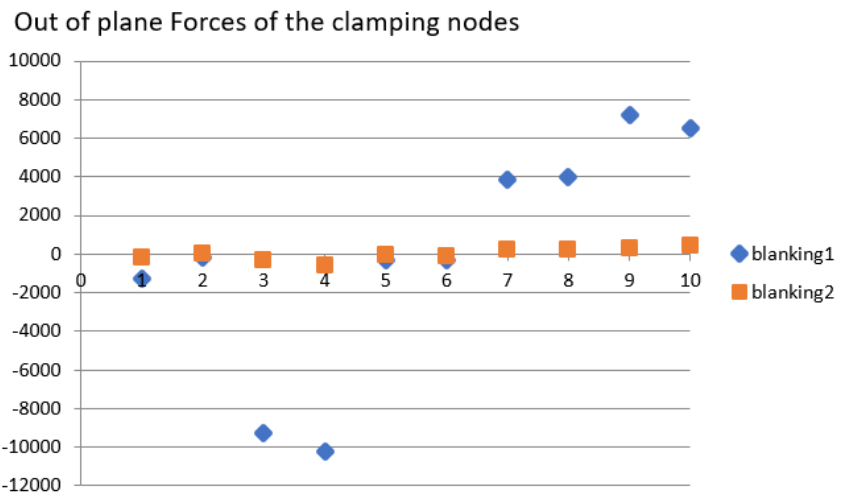

Figure 13 Calculation of out of plane clamping forces at node $1-10$

A more profound way to arrange for adaptive holding of the part that is subject for material removal would be to use a multi-axis and adaptively programmable machine tool. This application offers the possibility to be able to reach several sides of the billet within the same set-up without any intermediate re-clamping.

The deflections imposed by continuous release of residual stresses due to the ongoing material removal can under such conditions be handled to a very high degree of accuracy.

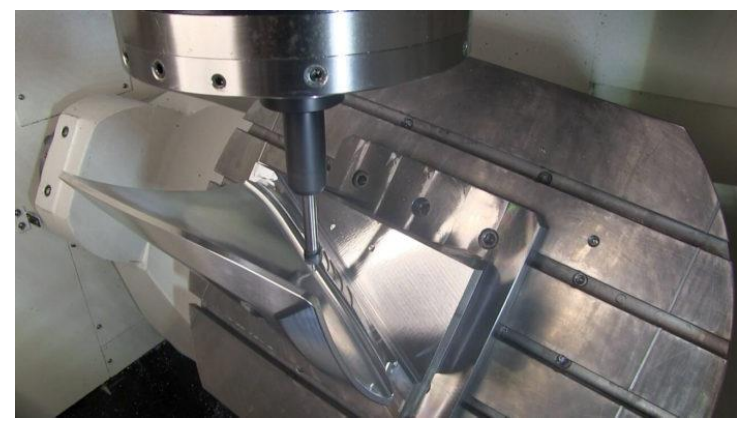

Figure 14 Multi axis machine tool configuration, Courtesy of Precise Tool and Manufacturing; Rochester, NY 14624

\subsection{Adjust tool path}

By taking into account the residual stresses during the tool path generation a significant improvement of geometrical accuracy can be achieved. A schematic overview of the steps required for adjusting the tool path using simulation is shown in fig. 15. 


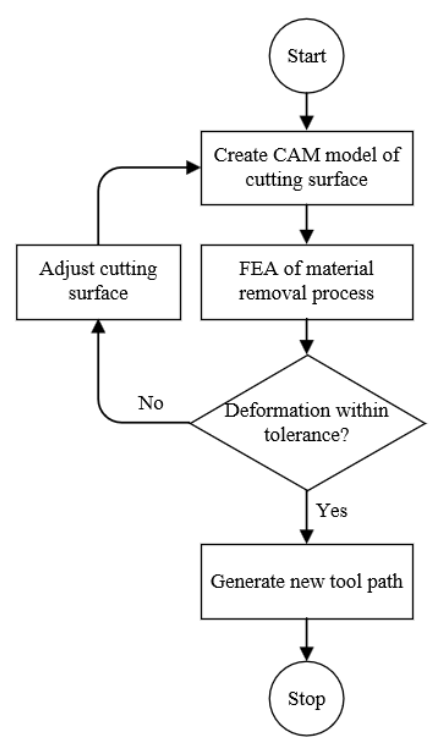

Figure 15 Schematic illustration of tool path optimization

The intended CAM cutting surfaces can be modeled with the help of CAD/CAM software. The generated 3D model will be used as input to the material removal simulation to predict the distortions. The input to the material removal simulation can be residual stresses generated with support from the Contour method. If the deformations are out of tolerances the cutting surface is adjusted. The loop is repeated until the deformations are within tolerances. A tool path that is adjusted due to the release of residual stresses is then generated. The concept is explained according to fig. 16 using an example with stress-free block exposed to clamping. The NC-code is prepared to machine a planar surface but after release of the clamping deviations in planarity will occur. By calculation of the deviations it is possible to adjust the NCCode by e.g. mirroring the deviations.

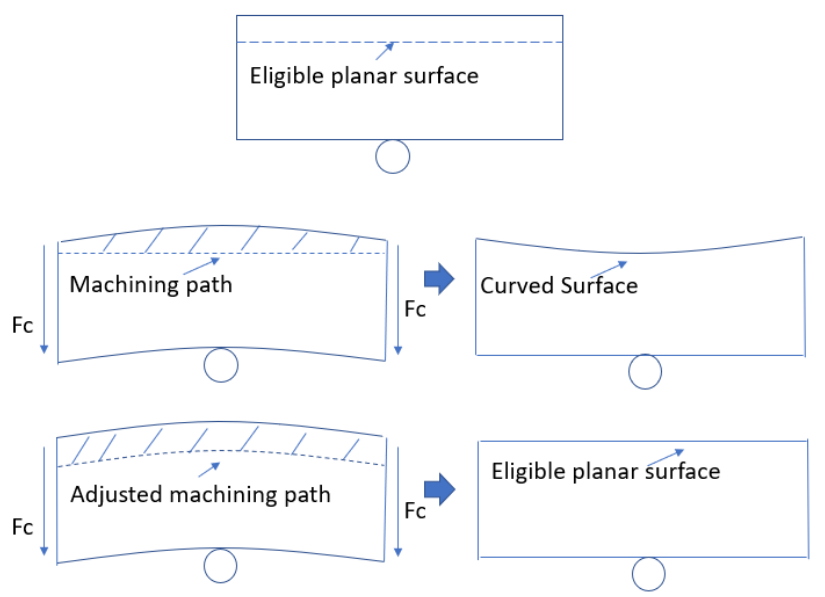

Figure 16 Schematic illustration of tool path compensation in a stress-free block exposed to clamping

However, the adjustment of one specific dimension may have consequences on other dimensions and tolerances. This is schematically illustrated with the example in fig. 17 using a block with internal residual stresses. Here the eligible planar machining top surface will be distorted due to the release of stresses during machining. This effect may be adjusted by preparing a modified machining path for the top side which adjust for the top surface deformations. However, the top side adjustment may also have effects on other parts of the geometry such as the bottom side geometry which in turn then must be adjusted. This may cause the top side to deform and the coupled problem may be solved using an iterative FEA adjustment strategy in order to create the two planar surfaces according to fig 18 .

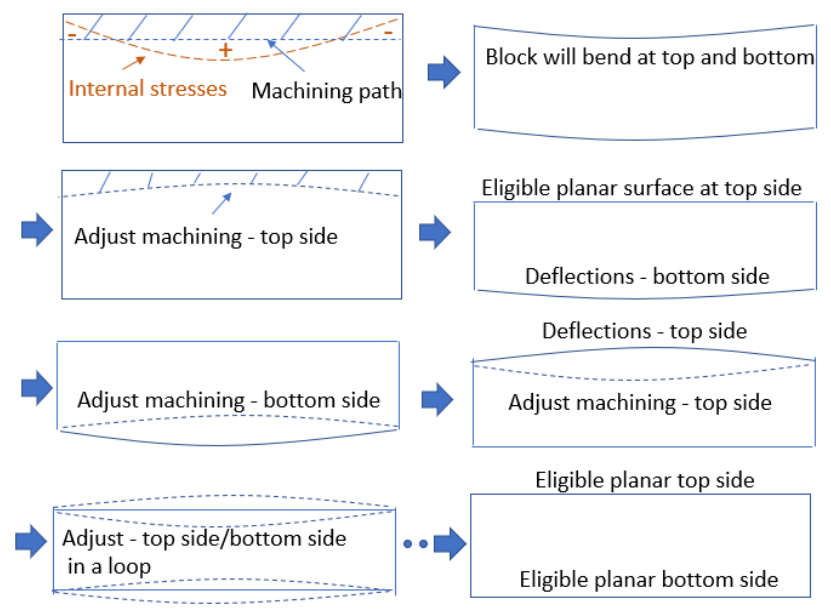

Figure 17 Schematic illustration of iterative tool path compensation in a block with internal stresses.

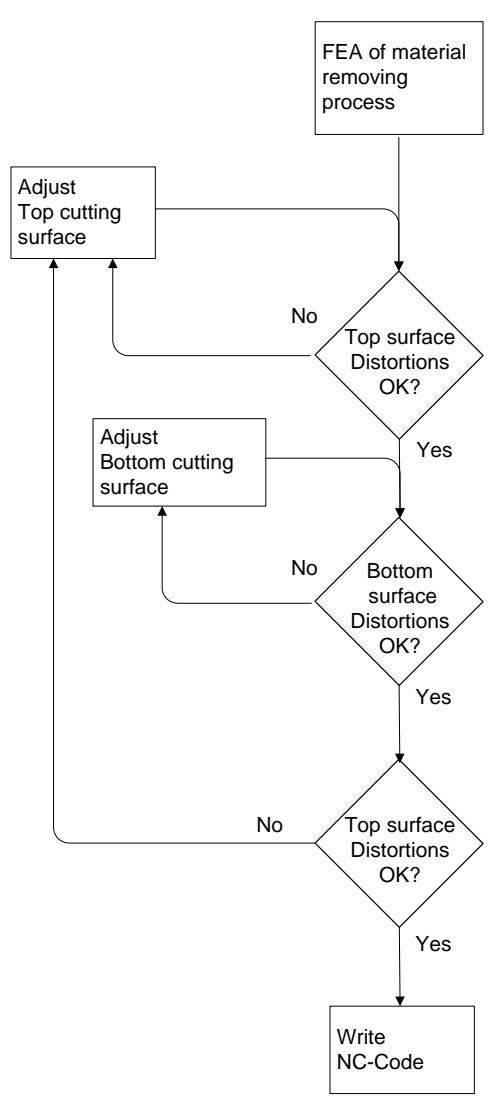

Figure 18 Adjustment strategy 
Comparison logic have to be built to compare the expected surface with simulated surface. If the distortions reside within the customer defined specifications, then adjusted NC code should be generated using CAM software.

The tool path optimization methodology requires successive selection of software applications, boundary conditions and application of initial stresses, thereby estimating the deformation arises during material removal. This will be run in an iterative manner involving CAD, CAM and FEA tools in order to minimize the distortion. The suggested adjustment should be evaluated carefully because the magnitude of distortion is a function of depth of cut, spring-back and cutting profile.

\section{Conclusions and future research}

This paper presents a methodology for minimizing distortions. The concept is a combination of procedures for prediction of machining distortions with procedures for adjustment of machining distortions. Machining distortion is a complex task and a close cooperation between several actors in the value chain may improve the possibilities to achieve good results. Issues for further research are discussed according to below.

Contour method - Sectioning: The sectioning must be done with high precision using e.g. EDM. However, EDM generates heat and leaves a thin re-cast layer on the cut surface. This effects the geometry and topology of the cut surface negatively and reduce the accuracy of the proceeding GOM- and FE analysis. Thus, the possibilities to increase the accuracy by replacing EDM with e.g. abrasive micro waterjet cutting should be investigated. The process is based on particle erosion without thermal impact on the sectioned surface. It shares the benefits of traditional waterjet cutting, whereas the smaller cutting system increase the precision 10 times cutting with $+/-0.01 \mathrm{~mm}$ in precision and $1 \mu \mathrm{m} \mathrm{Ra}$ value. The impact on surface topology is thereby minimal.

Contour method $-3 D$ stress prediction: In order to reduce the need for multiple cuts a numerical iterative approach for prediction of 3-dimensional stresses based on single cut measurements and FEM is proposed according to Section 3. The results for the test case indicate very were good results but further investigations are recommended.

Adaptive fixtures: During machining, the workpiece must be held and locked to a fixture which must be able to handle the clamping- and geometry changes that occur during the machining. Thus, further research is required in order to develop flexible adaptive clamping and support of the workpiece to the fixture in order to handle springback effects during machining.

Adjusting Tool Path: This paper presents a new concept for tool path optimization. Further research is required in order to analyse parts with complex surfaces and residual stress distributions and where several demands concerning geometry dimensions and tolerances from the customer has to be fulfilled.

\section{Acknowledgements}

The authors thank Vinnova that funded this research via the National Aeronautics Research Program (SMF-Flyg project), the SIP Produktion 2030 Program (PRIZE-project and CUBE project) and also Vastra Gotalands Regionen.

\section{References}

[1] Chatelain, Lalonde, Tahan, "A Comparison of the Distortion of Machined Parts Resulting From Residual Stresses Within Workpieces", Recent Advances in Manufacturing Engineering, ISBN: 9781-61804-031-J2

[2] Lequeu, Lassince, Warner, Raynaud, "Engineering for the future: Weight saving and cost reduction initiatives," Aircr. Eng. Aerosp. Technol., vol. 73, no. 2, pp. 147-159, 2001.

[3] Prime, Dewald, "The Contour Method" Chapter 5 in Practical Residual Stress Measurement Methods, Wiley-Blackwell, pp. 109-138Chapter," WileyBlackwell, Las Alamos, 2001.

[4] Johnsson, "Residual stress measurements using the Contour method", PhD thesis, University of Manchester School of materials, 2008 Manchester UK.

[5] Werke, Wretland, Ottosson, Holmberg, Machens, Semere, "Geometric distortion analysis using a combination of the contour method and machining simulation", 51st CIRP conference of Manufacturing Systems, 2018 Stockholm

[6] Mocellin, Cerutti, "Numerical prediction of distortions during machining of large aluminium aeronautical parts. Materwiss. Werksttech., vol. 47, no. 8, pp. 699-709, 2016.

[7] Guo, Zuo, Wang, Xu, Wang, Hu, "The application of FEM technology on the deformation analysis of the aero thin-walled frame shape workpiece," Key Eng. Mater., vol. 315-316, pp. 174-179, 2006.

[8] Cerutti, Mocellin, "Influence of the machining sequence on the residual stress redistribution and machining quality: Analysis and improvement using numerical simulations," Int. J. Adv. Manuf. Technol., vol. 83, no. 1-4, pp. 489-503, 2016.

[9] Chantzis, Van-Der-Veen, Zettler, Sim, “An industrial workflow to minimise part distortion for machining of large monolithic components in aerospace industry," in Procedia CIRP, 2013, vol. 8, pp. 281286. 
[10] Prime, Newborn, Balog, Quenching and cold work residual stresses in aluminum hand forgings: Contour method measurement and FEM prediction. Materials Science Forum 426-432 (2003) 435-440.

[11] DeWald, Hill, Multi-axial contour method for mapping residual Stresses in continuously processed bodies. Experimental Mechanics 46 (2006) 473-490.

[12] Borboni, Aggogeri, Merlo, Pellegrini, Amici, PKM Mechatronic Clamping Adaptive Device, International Journal of Advanced Robotic Systems, April 2015.

[13] Papastathis, Ryll, Bone, Ratchev, Development of a Reconfigurable Fixture for the Automated Assembly and Disassembly of High Pressure Rotors for RollsRoyce Aero Engines, IFIP AICT 315, pp. 283-289, 2010.

[14] Intelligent fixtures for the machining of low rigidity components, INTEFIX Report June 2016, EU Seventh Framework Programme (grant agreement no 609306). 